\title{
Delivery Channels to Close the Nutrient Intake Gap -What to Do without Data?
}

\author{
Regina MoEnch-PfAnNeR ${ }^{1}$ and Klaus KraEMER ${ }^{2}$ \\ ${ }^{1}$ The Global Alliance for Improved Nutrition (GAIN), Singapore \\ ${ }^{2}$ Sight and Life, Switzerland
}

\begin{abstract}
Summary It is widely documented that the intake of a micronutrient-dense food can be a challenge in environments where populations do not have sufficient purchasing power, full access to affordable nutritious foods, nutrition knowledge, or access to safe water, healthcare and hygienic living conditions. Micronutrient deficiencies during the critical 1000-day period have negative consequences for the cognitive and physical development of children, as well as longer-lasting effects on productivity and economic potential during adulthood. Accurate data on nutrition and health is largely lacking in many countries, which compromises the targeting of nutrition interventions to those in need. To overcome the problems of targeting the interventions, countries rely on the sketchy data that is available at the national level and lean also on international information systems. One of the efforts to stimulate greater investments and attention from policy- and decision-makers to eliminate micronutrient deficiencies in high-burden countries is the Hidden Hunger Index (HHI) launched in 2013 by Sight and Life, and recently updated to provide net changes from 19952011. The HHI and maps capture the magnitude of micronutrient deficiencies in countries and their contribution to the disease burden and social and economic development. These analyses are also critical for informing and tracking the impact of policy and programmatic efforts to reduce micronutrient deficiencies, and to advance the global and national nutrition agenda. Finally, our work highlights the lack of data available to measure and track micronutrient status. There is an urgent need to invest in (micronutrient) nutrition data collection to develop comprehensive information systems.
\end{abstract}

Key Words micronutrient-dense food, Hidden Hunger Index, nutrition data 